\title{
Assessing cognition and daily function in early dementia using the cognitive- functional composite: findings from the Catch-Cog study cohort
}

Roos J. Jutten ${ }^{1 *}$ (D), John E. Harrison ${ }^{1,2,3}$, Philippe R. Lee Meeuw Kjoe', Silvia Ingala ${ }^{1,4}$, R. Vreeswijk ${ }^{5}$, R. A. J. van Deelen ${ }^{5}$, Frank Jan de Jong ${ }^{6}$, Esther M. Opmeer ${ }^{7,8}$, André Aleman7, Craig W. Ritchie ${ }^{9}$, Philip Scheltens ${ }^{1}$ and Sietske A. M. Sikkes ${ }^{1,10}$

\begin{abstract}
Background: The cognitive-functional composite (CFC) was designed to improve the measurement of clinically relevant changes in predementia and early dementia stages. We have previously demonstrated its good test-retest reliability and feasibility of use. The current study aimed to evaluate several quality aspects of the CFC, including construct validity, clinical relevance, and suitability for the target population.
\end{abstract}

Methods: Baseline data of the Capturing Changes in Cognition study was used: an international, prospective cohort study including participants with subjective cognitive decline (SCD), mild cognitive impairment (MCI), Alzheimer's disease (AD) dementia, and dementia with Lewy bodies (DLB). The CFC comprises seven existing cognitive tests focusing on memory and executive functions (EF) and the informant-based Amsterdam Instrumental Activities of Daily Living Questionnaire (A-IADL-Q). Construct validity and clinical relevance were assessed by (1) confirmatory factor analyses (CFA) using all CFC subtests and (2) linear regression analyses relating the CFC score (independent) to reference measures of disease severity (dependent), correcting for age, sex, and education. To assess the suitability for the target population, we compared score distributions of the CFC to those of traditional tests (Alzheimer's Disease Assessment Scale-Cognitive subscale, Alzheimer's Disease Cooperative Study-Activities of Daily Living scale, and Clinical Dementia Rating scale).

Results: A total of 184 participants were included (age $71.8 \pm 8.4 ; 42 \%$ female; $n=14$ SCD, $n=80 \mathrm{MCl}, n=78 \mathrm{AD}$, and $n=12$ DLB). CFA showed that the hypothesized three-factor model (memory, EF, and IADL) had adequate fit $(\mathrm{CFI}=.931, \mathrm{RMSEA}=.091, \mathrm{SRMR}=.06)$. Moreover, worse CFC performance was associated with more cognitive decline as reported by the informant $(\beta=.61, p<.001)$, poorer quality of life $(\beta=.51, p<.001)$, higher caregiver burden $(\beta=-.51, p<.001)$, more apathy $(\beta=-.36, p<.001)$, and less cortical volume $(\beta=.34, p=.02)$. Whilst correlations between the CFC and traditional measures were moderate to strong (ranging from -.65 to .83 , all $p<.001$ ), histograms showed floor and ceiling effects for the traditional tests as compared to the CFC.

(Continued on next page)

\footnotetext{
* Correspondence: r.jutten@vumc.nl

${ }^{1}$ Alzheimer Center Amsterdam, Department of Neurology, Amsterdam

Neuroscience, Amsterdam UMC, Vrije Universiteit Amsterdam, Amsterdam,

The Netherlands

Full list of author information is available at the end of the article
}

(c) The Author(s). 2019 Open Access This article is distributed under the terms of the Creative Commons Attribution 4.0 International License (http://creativecommons.org/licenses/by/4.0/), which permits unrestricted use, distribution, and reproduction in any medium, provided you give appropriate credit to the original author(s) and the source, provide a link to the Creative Commons license, and indicate if changes were made. The Creative Commons Public Domain Dedication waiver (http://creativecommons.org/publicdomain/zero/1.0/) applies to the data made available in this article, unless otherwise stated. 
(Continued from previous page)

Conclusions: Our findings illustrate that the CFC has good construct validity, captures clinically relevant aspects of disease severity, and shows no range restrictions in scoring. It therefore provides a more useful outcome measure than traditional tests to evaluate cognition and function in $\mathrm{MCl}$ and mild $\mathrm{AD}$.

Keywords: Alzheimer's disease, Cognition, Composite, Daily function, Dementia, Construct validation, Instrumental activities of daily living, Mild cognitive impairment, Outcome measures

\section{Background}

Alzheimer's disease (AD) is the leading cause of dementia worldwide and has been the target of clinical trials and intervention studies for many years [1]. In the past decade, the research field has shifted towards earlier clinical stages of dementia and to predementia stages such as mild cognitive impairment (MCI) [2]. Remarkably, the selection of cognitive and functional outcome measures to evaluate treatment effects has not been adapted to the shift in treatment target populations. Measures originally designed for mild to severe dementia, such as the Alzheimer's Disease Assessment ScaleCognitive subscale (ADAS-Cog [3]), are still widely used as primary endpoints in MCI and early AD dementia trials. Several studies have shown that those older, traditional measures are insensitive to change over time in MCI and mild dementia $[4,5]$, as they focus on cognitive domains and everyday activities that are unaffected in those disease stages [5-7]. This limits their clinical relevance in the predementia window and leads to range restrictions in scoring (i.e., floor and ceiling effects) [4, 8]. Hence, researchers and regulatory agencies have expressed the urgent need for a sensitive measure that is capable of detecting clinically relevant changes at early clinical stages of AD [9-13]. The same holds for dementia with Lewy bodies (DLB), the second most common cause of dementia, of which both pathology and clinical manifestations show considerable overlap with $\mathrm{AD}[14,15]$.

The Capturing Changes in Cognition (Catch-Cog) project was initiated to fulfill the need for a sensitive, clinically relevant outcome measure for use in MCI and mild dementia. We designed a novel cognitive-functional composite (CFC) measure in our expert working group, basing our selections on previously published work and input from MCI and dementia patients and caregivers [16]. The resulting CFC consists of a short cognitive test battery focusing on memory and executive functioning (EF) [17], as these are the cognitive domains that have been shown to decline in predementia and early stages of dementia [13]. The rationale of specific cognitive tests included in this battery has been described in more detail elsewhere [16]. Briefly, the selection was based on empirical evidence on their sensitivity to change, as reflected by the absence of floor and ceiling effects in
MCI and mild AD [17]. To amplify its clinical relevance, we augmented the cognitive test battery with a previously validated everyday functioning measure: the Amsterdam Instrumental Activities of Daily Living Questionnaire (A-IADL-Q) [18]. The A-IADL-Q assesses the problems in cognitively complex everyday activities such as cooking, managing finances, and using technological devices $[19,20]$. In item response theory analyses, these activities were found to be the activities most sensitive to cognitive decline [21]. The A-IADL-Q was previously demonstrated to be sensitive to the decline in dementia, as well as able to capture difficulties in instrumental activities of daily living (IADL) functioning in MCI and subjective cognitive decline (SCD) [21, 22].

Combining these cognitive and functional measures into the CFC summarizes cognitive and everyday performance and may thereby provide a single clinically relevant score. Both the Food and Drug Association (FDA) and the European Medical Agency (EMA) encourage the use of such composite endpoints to evaluate novel drug therapies and interventions [11, 12]. These agencies also stipulate that composite measures should be (1) carefully designed, (2) validated in an independent prospective cohort study, and (3) "bear some relevance to existing tools for which historical experience exists" [12]. In the Catch-Cog study, we have sought to meet these criteria by performing an extensive construct validation of the CFC. In a previous report, we demonstrated that the CFC exhibits high test-retest reliability and good feasibility of use [23], which are pivotal prerequisites for a reliable and valid outcome measure [24]. Having demonstrated this, we embarked on a longitudinal construct validation in an independent prospective cohort across the clinical spectrum from SCD to mild dementia. The main aim of this study is to validate the CFC in MCI and mild $\mathrm{AD}$ dementia stages, but we will also explore whether the CFC could be of use in individuals with SCD and DLB.

In the current study, we performed a psychometric evaluation of the CFC using baseline data of the Catch-Cog study. We aimed to evaluate several quality aspects of the CFC, including the construct validity, clinical relevance, and suitability for the target population. Therefore, we investigated the CFC's factor structure and compared the CFC score with reference measures of disease severity such as informant reports and global 
cortical atrophy [16]. We also examined CFC score distributions in direct comparison to currently used tests, including the ADAS-Cog [3], the Clinical Dementia Rating scale Sum of Boxes (CDR-SB) [25], the Alzheimer's Disease Cooperative Study-Activities of Daily Living scale (ADCS-ADL) [26], and the Alzheimer's Disease Composite Score (ADCOMS) procedure [27].

\section{Methods}

\section{Study design and participants}

In this cross-sectional study, we employed baseline data from the Catch-Cog study, which is an international, multicenter, prospective cohort study. Participants $(N=184)$ were recruited via the (1) Alzheimer Center Amsterdam, Amsterdam UMC, location VU University Medical Center, The Netherlands $(n=102)$; (2) Alzheimer Center Erasmus Medical Center (EMC, $n=14$ ), Rotterdam, The Netherlands; (3) University Medical Center Groningen (UMCG, $n=39$ ), The Netherlands; and (4) the Centre for Dementia Prevention, Edinburgh, Scotland $(n=29)$. We recruited participants who met the research criteria for SCD [28], the clinical criteria for $\mathrm{MCI}$ due to $\mathrm{AD}$ [2], probable $\mathrm{AD}$ dementia [29], or DLB dementia [15]. Other inclusion criteria were (1) Mini-Mental State Examination (MMSE) score $\geq 18$, (2) age $\geq 50$, and (3) availability of a study partner who was able and willing to participate. Exclusion criteria were (1) presence of any other neurological disorder, (2) presence of a major psychiatric disorder such as severe personality disorder or depression (Geriatric Depression Scale score $\geq 6$ [30]), (3) current abuse of alcohol and/or drugs, and (4) simultaneously participating in a clinical trial.

Before inclusion, participants had undergone a standard diagnostic work-up in their study center, including at least medical history, neurological examination, and cognitive assessment. Structural brain imaging was available for a subset of the study cohort. Diagnoses were performed during a multidisciplinary consensus meeting, containing at least a neurologist or psychiatrist and with neuropsychology input. In the UMCG, SCD, and MCI, participants were also recruited via advertisements in local newspapers. After responding to this advertisement, eligible participants were screened by a neuropsychologist and neurologist to investigate whether they met the criteria for SCD or MCI [28].

The Medical-Ethical Committee of the VU University Medical Center approved the study for all Dutch centers. The South East Scotland Research Ethics Committee approved the study for the Scottish site. All participants and study partners provided written and oral informed consent.

\section{The cognitive-functional composite Cognitive component}

The cognitive test battery of the CFC included the three ADAS-Cog memory subscales Word Recognition, Word
Recall and Orientation [3]; the Controlled Oral Word Association Test (COWAT); category fluency test (CFT); Digit Span Backward (DSB) and Digit Symbol Substitution Test (DSST) [31]. During the word recognition test, the participant is required to learn a list of 12 words and identify these words when mixed among 12 other distracter words (one point for each incorrect response, score range 0-12). During word recall, the participant is given three trials to learn a list of ten high-imagery nouns (total score entails the average number of words not recalled across the three trials, score range $0-10$ ). The orientation subtest includes eight questions regarding the participant's orientation to person, place, and time (one point for each incorrect response, score range $0-8$ ). The COWAT assesses the participant's phonemic fluency skills using the letters D-A-T in The Netherlands or F-A-S in English and a total time of $60 \mathrm{~s}$ per letter (one point for each correct non-repeated word). The CFT examines the participant's semantic fluency by requiring them to generate as many exemplars of the category animals within $60 \mathrm{~s}$ (one point for each correct unique animal). The DSB requires the participant to reproduce sequences of digits of increasing length in the reversed order (score range $0-12$ ). The DSST is a timed EF test during which participants have to substitute as many digits by unique geometric symbols within $90 \mathrm{~s}$ (one point for each correct substituted symbol).

\section{Functional component}

The functional component consisted of the short version of the A-IADL-Q [21]. The A-IADL-Q is a computerized, informant-based questionnaire covering a broad range of complex IADL [19]. The short version consists of 30 items covering household, administration, work, computer use, leisure time, appliances, and transport activities. For each item, difficulty in performance is rated on a 5-point Likert scale (ranging from "no difficulty in performing this task" to "no longer able to perform this task"). Scoring is based on item response theory, a paradigm linking item responses to an underlying latent trait [32]. This results in a latent trait score ( $z$-score), reflecting one's level of IADL functioning, with higher scores indicating better IADL functioning [21].

\section{CFC scoring}

To create CFC scores, the directionality of the three ADAS-Cog subtest scores were reversed, so that higher scores reflected better performance. Subsequently, all cognitive subtest scores were transformed into $z$-scores with total group mean and standard deviation (SD) as reference values. The cognitive composite was computed as a weighted $z$-score of all seven cognitive subtests, whereas the functional component score was the A-IADL-Q score. The overall CFC composite score was 
computed as a weighted $z$-score of the cognitive composite and A-IADL-Q, with higher scores indicating better performance.

\section{Reference measures Traditional tests of cognition and function}

Traditional tests to compare the CFC with included the MMSE, ADAS-Cog-13, ADCS-ADL, and CDR-SB. The MMSE is a global cognitive screening test, with a total score ranging from 0 to 30 and higher scores reflecting better performance [33]. The ADAS-Cog-13 yields a measure of cognitive performance by combining ratings of 13 subtests (e.g., word lists recognition and recall, constructional praxis, object and finger naming). Total scores range from 0 to 85 , with higher scores indicating more severe impairment [3]. The ADCS-ADL assesses the functional abilities affected in mild-to-moderate AD. For 23 different basic and instrumental activities, the levels of performance and independency during the past 4 weeks were rated by the study partner. Total scores range from 0 (non-performance or need for extensive help) to 78 (independent performance) [26]. The CDR has been developed for the staging of dementia severity. The participant's cognitive and functional performance is rated in 6 areas: memory, orientation, judgment and problem solving, community affairs, home and hobbies, and personal care. Each area is rated as 0 (healthy), 0.5 (questionable dementia), 1 (mild dementia), 2 (moderate dementia), or 3 (severe dementia). Adding the rating of all boxes results in a total CDR-SB score ranging from 0 to 18 , with higher scores reflecting severe dementia [25, 34]. The ADCOMS is a recently designed, statistically derived composite scoring procedure, consisting of two MMSE items ("orientation to time" and "copy design"), 4 ADAS-Cog subtests (delayed word recall, orientation, word recognition, and word recall) and all 6 CDR-SB subscores. All items are differentially weighted yielding a score ranging from 0 to 1.27 with higher scores implying greater impairment [27].

\section{Reference measures of disease severity}

Informant reports of disease severity included the Cognitive Function Instrument study partner version (CFI-SP) [35], Quality of Life in Alzheimer's Disease (QoL-AD) [36], the short version of Zarit Burden Inventory (ZBI-12) [37], and the Apathy Evaluation Scale (AES) [38]. The CFI-SP includes 14 items on a decline in day-to-day cognitive and functional abilities compared to 1 year ago. Response options include "yes" (0), "no" (1), or "maybe" (0.5), with total scores ranging from 0 to 14. The QoL-AD consists of 13 items, rated on a 4-point scale. Total scores range from 13 to 52, with higher scores reflecting better quality of life. The ZBI is one of the most commonly used instruments for assessing the aspects of caregiver burden [37]. Each item was rated on a 5-point scale, with total scores ranging from 0 to 60 and higher scores suggesting greater caregiver burden. The AES consists of 18 statements about the participant's thoughts, feelings, and activity, which are rated on a 4-point scale. Total scores range from 0 to 72 , with higher scores indicating more severe apathy.

Magnetic resonance (MR) images were acquired locally at each center in $3 \mathrm{~T}$ scanners. A minimum acceptable protocol was approved and then optimized at each site due to scanner differences (see Additional file 1). The images were checked for quality by an experienced rater. Volumetric measurements were processed on 3D T1-weighted (3DT1) images with Statistical Parametric Mapping 12 (SPM12) software (Wellcome Trust Centre for Neuroimaging, University College London, UK) running in MATLAB 2011a (MathWorks Inc., Natick, MA, USA). Prior to processing, the origin in each scan was manually set to the anterior commissure. Scans were segmented into gray matter (GM), white matter (WM), and cerebrospinal fluid (CSF). Total GM (i.e., the sum of all GM voxels) and total intracranial volume (TIV) (i.e., the sum of GM, WM, and CSF volumes) were derived from the segmented images in native space (units in liter). Cortical volume was defined as the total GM volume normalized for head size, divided by TIV.

\section{Procedures}

Study visits took place at the hospital or the participant's home, depending on the participant's preference. A trained rater assessed the cognitive tests according to standardized instructions, starting with the MMSE and followed by the cognitive part of the CFC (word recognition, orientation, CFT, COWAT, DSST, DSB, word recall) and the remaining ADAS-Cog-13 tests. In the meantime, the study partner completed the A-IADL-Q, ZBI, and QoL-AD independently on an iPad. Subsequently, the participant completed the QoL-AD on the iPad with assistance from the rater. Finally, the rater completed the ADCS-ADL and CDR interview with the study partner. The total duration of a complete assessment was approximately $90 \mathrm{~min}$. A shortened protocol was used in the SCD and DLB participants, as it was not our purpose to compare the CFC to the traditional tests that were not designed to assess the progression in these groups. Therefore, SCD and DLB participants only underwent the MMSE and cognitive battery of the CFC whilst their study partner completed the A-IADL-Q.

\section{MRI procedures}

MR scans acquired less than 6 months prior to the study visit were available for a subset of the study cohort. These included at least 3D T1- and T2-weighted imaging (T2) and 3D fluid-attenuated inversion recovery (FLAIR). Participants without a recent MRI scan available but who 
agreed to undergo a structural MRI scan were also scanned at $3 \mathrm{~T}$ with the same structural sequences which took about $30 \mathrm{~min}$.

\section{Statistical analyses}

Statistical analyses were performed using SPSS version 22.0 (IBM Corp., Armonk, NY) and R Studio (R Core Team, 2018). Statistical significance level was set at $p$ value $<.05$, unless otherwise indicated. Demographic and clinical differences between the groups were investigated using chi-square tests, one-way analyses of variance (ANOVA) followed by Hochberg's post hoc tests, and independent $t$ tests for measures only available for the $\mathrm{MCI}$ and $\mathrm{AD}$ group.

\section{Construct validity and clinical relevance}

We performed confirmatory factor analyses (CFA) including all CFC subtests to investigate the CFC's underlying factor structure. We evaluated a single-factor, two-factor (memory and $\mathrm{EF}$ ), and three-factor (memory, EF, and IADL) model. In the two-factor model, the memory factor included the word recognition, orientation, and word recall tests and the EF factor included the CFT, COWAT, DSST, DSB, and A-IADL-Q. The three-factor model had a similar memory and EF factor, except that the A-IADL-Q was excluded from the EF factor and included a separate factor. We compared these models using chi-square tests and by evaluating their Comparative Fit Index (CFI), root mean squares of error approximation (RMSEA), and standardized root mean square residual (SRMR) indices, with $\mathrm{CFI} \geq .90$, RMSEA $<.08$, and SRMR $<.08$ considered as adequate fit [39]. We hypothesized that the three-factor model would fit, based on preparatory work on the cognitive component showing two underlying factors [40], and A-IADL-Q reflecting one underlying factor [19]. As a sensitivity analysis, all aforementioned CFA model evaluations were repeated in a restricted sample of $\mathrm{MCI}$ and mild AD participants, as this was the primary target population of the CFC.

Next, we investigated the differences in CFC scores across diagnostic groups using ANOVA followed by Hochberg's post hoc tests, to examine whether scores would decrease from SCD to dementia. We assessed the association between the CFC variables and reference measures of disease severity, by performing linear regression analyses for each reference measure (CFI-SP, QoL-AD, ZBI-12, and AES, as dependent) and CFC score, age, sex and education as independents. To evaluate the added clinical value of the A-IADL-Q, we also investigated a second model including the cognitive component score and A-IADL-Q score as separate independents. The association between CFC score and gray matter volume was assessed with a linear regression analysis correcting for age, sex, years of education, and scanner type. We computed Pearson's correlation coefficients to investigate the relation between CFC scores and traditional cognitive and functional measures.

\section{Quality for the target population}

As the CFC was initially designed for $\mathrm{MCI}$ and mild $\mathrm{AD}$ dementia, the comparison analyses with traditional measures and ADCOMS were performed using the $\mathrm{MCI}$ and $\mathrm{AD}$ groups only. Using this sample, we compared histograms of score distributions of the CFC, traditional tests, and ADCOMS to inspect range restrictions in scoring. To allow for appropriate comparisons between the CFC components and traditional tests, the histograms for the ADAS-Cog, ADCS-ADL, and CDR-SB score distributions were based on the standardized scores. Additionally, we reported original score ranges and distribution parameters (percentiles, skewness, and kurtosis) for all tests.

\section{Results}

\section{Descriptive characteristics}

The total sample $(N=184)$ had a mean age of $71.8 \pm 8.4$ years, $42 \%$ were female, and mean years of education was $13.6 \pm 3.8$. The majority had a diagnosis of MCI $(n=80)$ or $\mathrm{AD}$ dementia $(n=78)$. Table 1 presents the demographic and clinical characteristics separately for each diagnostic group. Groups differed in terms of age $(F=2.99, p=.033)$, but there were no significant differences regarding sex and education. MMSE scores were lower for dementia (AD, 24.0 \pm 3.3 ; DLB, $24.8 \pm 3.1)$ compared to $\mathrm{MCI}(26.7 \pm 2.3)$ and SCD $(29.3 \pm 1.2)$. The AD group also performed worse on the ADAS-Cog (mean difference 6.3 points, $p<.001$ ), ADCS-ADL (mean difference 3.8 points, $p=.01$ ) and CDR-SB (mean difference 2.4 points, $p<.001$ ) when compared to the $\mathrm{MCI}$ group. Study partners of AD participants reported worse CFI-SP scores (mean difference 2 points, $p<.001$ ), lower quality of life scores (mean difference 2.2 points, $p=.026$ ), higher caregiver burden (mean difference 3.7 points, $p=.004$ ), and higher apathy levels (mean difference 3.4 points, $p=.039$ ) compared to study partners of MCI participants.

\section{Construct validity and clinical relevance}

CFA showed that the hypothesized three-factor model including memory, EF, and IADL had an adequate fit $(\mathrm{CFI}=.931, \quad \mathrm{RMSEA}=.091 \quad(90 \% \mathrm{CI}=.058-.124 \quad$ and SRMR $=.06$ ), although the RMSEA index did not reach the predefined cutoff $(<.08)$. The three-factor model (Fig. 1) described the data better than the single- or two-factor models (Table 2). Similar results were found after repeating the analyses in a restricted sample of $\mathrm{MCI}$ and mild AD participants (CFI $=.918$, RMSEA $=.09$ $(90 \% \mathrm{CI}=.053-.122$ and $\mathrm{SRMR}=.06)$.

As expected, overall CFC scores decreased concomitantly to progression across the spectrum from SCD 
Table 1 Descriptive characteristics and test scores separately for each diagnostic group

\begin{tabular}{|c|c|c|c|c|c|c|}
\hline & $\operatorname{SCD}(n=14)$ & $\mathrm{MCl}(n=80)$ & $\mathrm{AD}(n=78)$ & $\operatorname{DLB}(n=12)$ & $p$ value & Post hoc between group comparisons* \\
\hline \multicolumn{7}{|l|}{ Descriptives } \\
\hline Age & $68.1(6.6)$ & $73.6(8.0)$ & $71.3(9.1)$ & $68.3(6.1)$ & .033 & Non-significant \\
\hline Female (\%) & $8(57.1 \%)$ & $29(36.3 \%)$ & $39(50 \%)$ & $2(16.7 \%)$ & .060 & N.A. \\
\hline Education & $15.2(4.8)$ & $13.7(3.6)$ & $13.2(4.0)$ & $13.6(3.3)$ & .36 & N.A. \\
\hline MMSE & $29.3(1.2)$ & $26.7(2.3)$ & $24.0(3.3)$ & $24.4(2.9)$ & $<.001$ & $\mathrm{SCD}>\mathrm{MCl}>\mathrm{AD} ; \mathrm{SCD}>\mathrm{DLB}$ \\
\hline \multicolumn{7}{|l|}{ CFC components } \\
\hline Cognitive composite & $.88(.50)$ & $.19(.50)$ & $-.31(.61)$ & $-.35(.71)$ & $<.001$ & $\mathrm{SCD}>\mathrm{MCl}>\mathrm{AD}, \mathrm{SCD}>\mathrm{DLB}, \mathrm{MCl}>\mathrm{DLB}$ \\
\hline A-IADL-Q & $.91(.79)$ & $.39(.73)$ & $-.48(.84)$ & $-.65(.90)$ & $<.001$ & $\mathrm{SCD}>\mathrm{AD} ; \mathrm{MCl}<\mathrm{AD} ; \mathrm{SCD}>\mathrm{DLB} ; \mathrm{MCl}>\mathrm{DLB}$ \\
\hline CFC score & $.89(.57)$ & $.29(.51)$ & $-.39(.61)$ & $-.51(.75)$ & $<.001$ & $\mathrm{SCD}>\mathrm{MCl}>\mathrm{AD} ; \mathrm{SCD}>\mathrm{DLB} ; \mathrm{MCl}>\mathrm{DLB}$ \\
\hline \multicolumn{7}{|l|}{ Reference measures } \\
\hline CFI-SP & N.A. & $6.9(3.2)$ & $4.9(2.8)$ & N.A. & $<.001$ & N.A. \\
\hline QoL-AD & N.A. & $33.8(5.3)$ & $31.6(5.2)$ & N.A. & .026 & N.A. \\
\hline ZBI-12 & N.A. & $10.7(7.7)$ & $14.4(7.9)$ & N.A. & .004 & N.A. \\
\hline AES & N.A. & $39.9(10.5)$ & $43.3(10.0)$ & N.A. & .053 & N.A. \\
\hline \multicolumn{7}{|l|}{ Traditional tests } \\
\hline ADAS-Cog & N.A. & $22.2(6.8)$ & $28.5(7.6)$ & N.A. & $<.001$ & N.A. \\
\hline ADCS-ADL & N.A. & $67.5(7.8)$ & 63.7 (8.8) & N.A. & .010 & N.A. \\
\hline CDR-SB & N.A. & 2.7 (1.9) & $5.1(2.2)$ & N.A. & $<.001$ & N.A. \\
\hline
\end{tabular}

Abbreviations: ADAS-Cog Alzheimer's Disease Assessment Scale-Cognitive subscale, ADCS-ADL Alzheimer's Disease Cooperation Study-Activity of Daily Living, AES Apathy Evaluation Scale, A-IADL-Q Amsterdam IADL Questionnaire, CDR-SB Clinical Dementia Rating Sum of Boxes, CFC cognitive-functional composite, CFI-SP Cognitive Function Instrument study partner version, MMSE Mini-Mental State Examination, QoL Quality of life, ZBI Zarit Burden Inventory

*Based on Hochberg's post hoc tests

$(.89 \pm .57)$ to MCI $(.29 \pm .51)$ and to AD or DLB dementia $(\mathrm{AD},-.39 \pm .61 ; \mathrm{DLB},-.52 \pm .75)$, with significant differences between all groups except the two dementia groups (AD and DLB). A similar pattern was found for the cognitive composite score. A-IADL-Q scores were significantly lower for $\mathrm{AD}$ as compared to SCD and MCI, as well as for DLB compared to MCI and SCD (Table 1). Figure 2 visualizes the decreased scores across the diagnostic groups, with the cognitive composite divided into a memory and EF score according to the CFA results. It also shows that the $\mathrm{CFC}$ score is similar for the two dementia groups and that in $\mathrm{AD}$, this score is driven by the memory factor rather than the EF factor, whereas the opposite is observed in DLB.

Lower CFC scores were associated with worse cognitive functioning as reported by the informant (corrected $\beta=.61, p<.001$ ), quality of life (corrected $\beta=.51, p<.001$ ), caregiver burden (corrected $\beta=-.51, p<.001$ ), and apathy level (corrected $\beta=-.36, p<.001)$. Regression models including the cognitive composite and A-IADL-Q as separate scores demonstrated the added clinical value of the A-IADL-Q (Table 3). We found moderate-to-strong associations between the cognitive composite and ADAS-Cog $(r=-.83 ; 95 \% \quad \mathrm{CI}=-.87$ to -.77$)$, the A-IADL-Q and ADCS-ADL $(r=.65 ; 95 \% \mathrm{CI}=.54-.73)$, and CFC score and CDR-SB $(r=-.69 ; 95 \% \mathrm{CI}=-.77$ to -.56$)$.
Brain MR images were available for 70 participants $(n=7$ $\mathrm{SCD}, n=27 \mathrm{MCI}$, and $n=36 \mathrm{AD}$ ). Linear regression analyses showed a significant association between normalized GM volume and CFC score (corrected $\beta=.34, p=.01$, Fig. 3), indicating that worse performance on the CFC was related to less cortical volume independently of age, sex, education, and scanner type.

\section{Quality for the target population}

Histograms including the total MCI and AD sample $(n=158)$ showed expected floor and ceiling effects in scoring for all the traditional measures (Fig. 4). Range restrictions were especially apparent for the ADASCog and ADCS-ADL (Table 4). For example, for the ADCS-ADL, 35\% of the participant scores were at the maximum end of the scale (between 70 and 78). By comparison, all CFC components showed normal distributions without range restrictions in scoring. Figure 5 displays a direct comparison between the ADCOMS and CFC score distributions, separately for the $\mathrm{MCI}$ and mild AD dementia group. Despite strong correlations between the ADCOMS and CFC scores $(r=-.76 ; 95 \% \mathrm{CI}=-.82$ to -.68$)$, it can be seen that the ADCOMS is more influenced by ceiling effects in scoring, particularly in the MCI subgroup. 


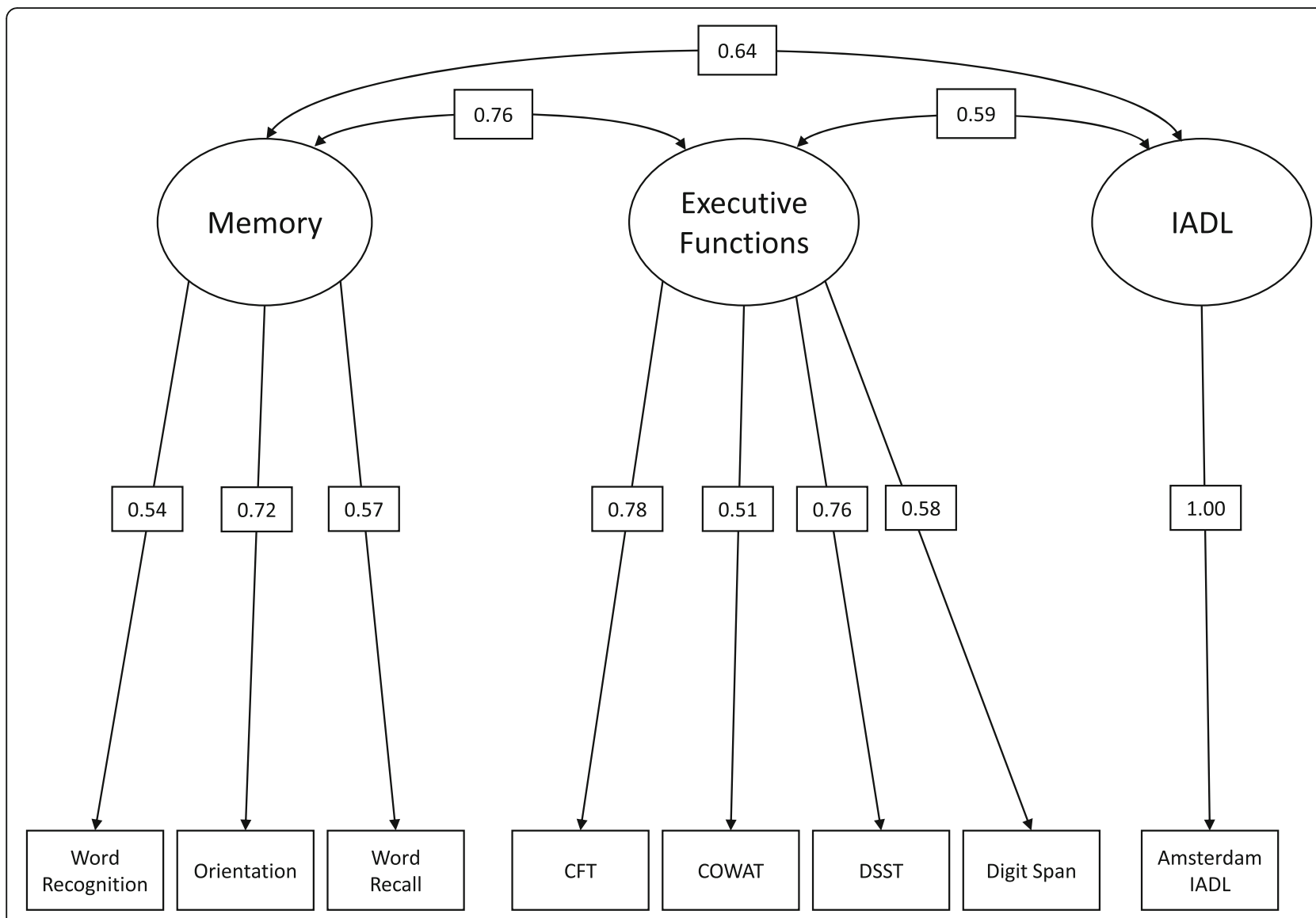

Fig. 1 Path diagrams showing the three-factor structure of the CFC, including the covariance between domains and variables

\section{Discussion}

We performed a psychometric evaluation of the novel CFC. Factor analyses confirmed the underlying structure of the CFC, reflecting the domain memory, EF, and IADL. The associations that we found between the CFC score and reference measures of disease severity further supported the construct validity and clinical relevance of the CFC. We also demonstrated that the CFC scores yielded fewer range restrictions in scoring as compared to traditional tests of cognition and function, indicating a better quality for the target population.
Construct validity, clinical relevance, and suitability for the target population are important quality aspects for clinical outcome measures [41]. Construct validity refers to whether an instrument measures what it intends to measure [24]. As a first step to assess this, we performed a CFA which confirmed that our novel combination of tests can be described by a memory, EF, and IADL component. Interestingly, we observed that the two cognition factors contributed differentially to the overall CFC score as seen in $A D$ versus $D L B$, with the $A D$ group scoring worse on the memory component compared to

Table 2 Fit statistics for confirmatory factor analysis models

\begin{tabular}{llllllllll}
\hline & Chi-square & df & $p$ value & CFI & RMSEA [90\%Cl] & SRMR & $\begin{array}{l}\text { Comparison with } \\
\text { single-factor model }\end{array}$ & $\begin{array}{l}\text { Comparison with } \\
\text { two-factor model }\end{array}$ \\
\hline Model 1: single factor & 62.952 & 20 & $<.001$ & .891 & $.108[.079-.139]$ & .070 & N.A. & N.A. & \\
Model 2: MEM + EF & 52.543 & 19 & $<.001$ & .915 & $.098[.067-.130]$ & .064 & ChiSq(1)=10.409, $p=.001$ & N.A. \\
Model 3A: MEM + EF + IADL & 45.269 & 18 & $<.001$ & .931 & $.091[.058-.124]$ & .060 & Chisq $(2)=17.683, p<.001$ & Chisq(1)=15.873, $p<.001$
\end{tabular}

Model 1: Single factor based on all eight CFC subtests

Model 2: MEM = Word Recognition + Orientation + Word Recall, EF = Controlled Word Association Test + Category Fluency + Digit Symbol Substitution + Digit Span Backward + Amsterdam IADL Questionnaire

Model 3: MEM = Word Recognition + Orientation + Word Recall, EF = Controlled Word Association Test + Category Fluency + Digit Symbol Substitution + Digit Span Backward, IADL = Amsterdam IADL Questionnaire

Abbreviations: CFI Comparative Fit Index, EF executive functioning, IADL instrumental activities of daily living; MEM memory, RMSEA Root mean squares of error approximation, SRMR Standardized root mean square residual 

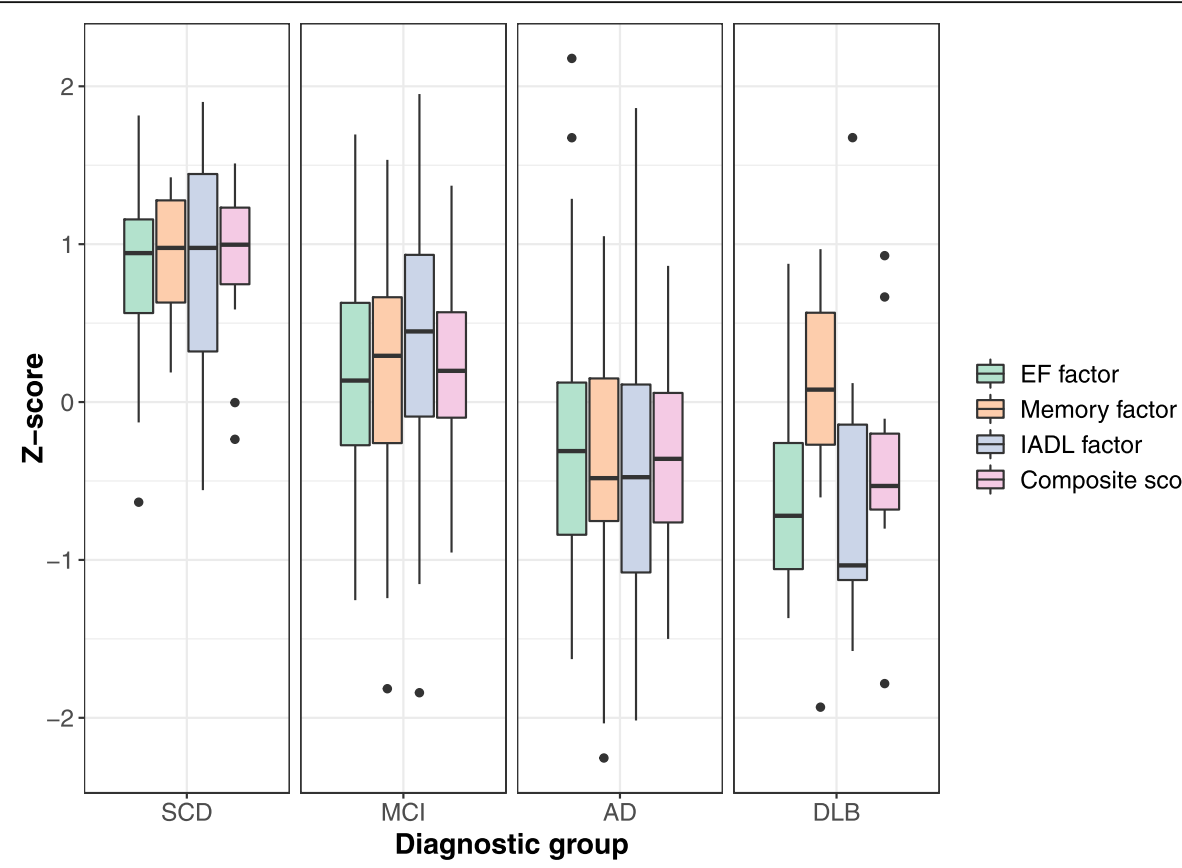

宁 IADL factor

官 Composite score

diagnostic group

the EF component and the DLB group vice versa. Notably, this is in line with the clinical pictures of both diseases with memory problems as the most prominent symptoms of early $\mathrm{AD}$ as opposed to more predominant EF problems in early DLB [14]. These results further confirm that the CFC score is an adequate reflection of the dimensionality of the construct to be measured.

Another aspect of construct validity is testing whether scores on an instrument are associated with scores on instruments that measure a similar construct [24]. We found moderate-to-strong associations with the traditional measures of cognition and function, which supports the construct validity of the CFC. It should, however, be noted that cognitive composite included three ADAS-Cog measures, which probably accounts for the strong association we found between these measures. We also demonstrated that the CFC is associated with other clinically relevant measures of disease severity, such as cognitive decline as reported by the informant, quality of life, caregiver burden, and apathy. Additionally, the association between CFC score and GM volume, which has shown to be a good biomarker of neurodegeneration in $\mathrm{AD}[42,43]$,

Table 3 Beta coefficients obtained from linear regression analyses relating CFC components to reference measures of disease severity

\begin{tabular}{|c|c|c|c|c|c|c|c|c|c|c|c|c|c|c|c|c|}
\hline & \multicolumn{4}{|c|}{ CFI-SP } & \multicolumn{4}{|c|}{ QoL-AD } & \multicolumn{4}{|c|}{ ZBI-12 } & \multicolumn{4}{|l|}{ AES } \\
\hline & \multirow[t]{2}{*}{ Beta } & \multicolumn{2}{|c|}{$95 \% \mathrm{Cl}$} & \multirow[t]{2}{*}{$p$ value } & \multirow[t]{2}{*}{ Beta } & \multicolumn{2}{|c|}{$95 \% \mathrm{Cl}$} & \multirow[t]{2}{*}{$p$ value } & \multirow[t]{2}{*}{ Beta } & \multicolumn{2}{|c|}{$95 \% \mathrm{Cl}$} & \multirow[t]{2}{*}{$p$ value } & \multirow[t]{2}{*}{ Beta } & \multicolumn{2}{|c|}{$95 \% \mathrm{Cl}$} & \multirow[t]{2}{*}{$p$ value } \\
\hline Model 1 & & & & & & & & & & & & & & & & \\
\hline CFC score & .61 & .48 & .74 & $<.001$ & .51 & .37 & .65 & $<.001$ & -.51 & -.65 & -.37 & $<.001$ & -.36 & -.51 & -.20 & $<.001$ \\
\hline Age & .14 & .02 & .27 & .028 & -.06 & -.20 & .08 & .388 & -.08 & -.21 & .06 & .280 & -.01 & -.16 & .14 & .913 \\
\hline Sex & .21 & .08 & .34 & .002 & .10 & -.04 & .24 & .171 & -.19 & -.33 & -.05 & .009 & -.19 & -.34 & -.04 & .014 \\
\hline Education & -.04 & -.17 & .09 & .578 & -.13 & -.27 & .02 & .087 & .20 & .06 & .34 & .006 & .05 & -.10 & .21 & .507 \\
\hline \multicolumn{17}{|l|}{ Model 2} \\
\hline Cognitive composite & -.09 & -.24 & .06 & .226 & -.10 & -.26 & .07 & .254 & -.02 & -.19 & .15 & .815 & -.01 & -.20 & .18 & .915 \\
\hline A-IADL-Q & .72 & .58 & .86 & $<.001$ & .62 & .46 & .77 & $<.001$ & -.52 & -.68 & -.36 & $<.001$ & -.37 & -.55 & -.19 & $<.001$ \\
\hline Age & .11 & -.01 & .23 & .066 & -.09 & -.22 & .04 & .182 & -.06 & -.19 & .08 & .409 & .01 & -.14 & .16 & .945 \\
\hline Sex & .18 & .06 & .30 & .004 & .07 & -.06 & .20 & .305 & -.17 & -.31 & -.03 & .017 & -.18 & -.33 & -.03 & .021 \\
\hline Education & .04 & -.09 & .16 & .552 & -.06 & -.20 & .08 & .405 & .16 & .02 & .30 & .030 & .02 & -.14 & .18 & .791 \\
\hline
\end{tabular}

Abbreviations: AES Apathy Evaluation Scale, A-IADL-Q Amsterdam IADL Questionnaire, CFC cognitive-functional composite, CFI-SP Cognitive Function Instrument study partner version, QoL Quality of life, ZBI Zarit Burden Inventory 


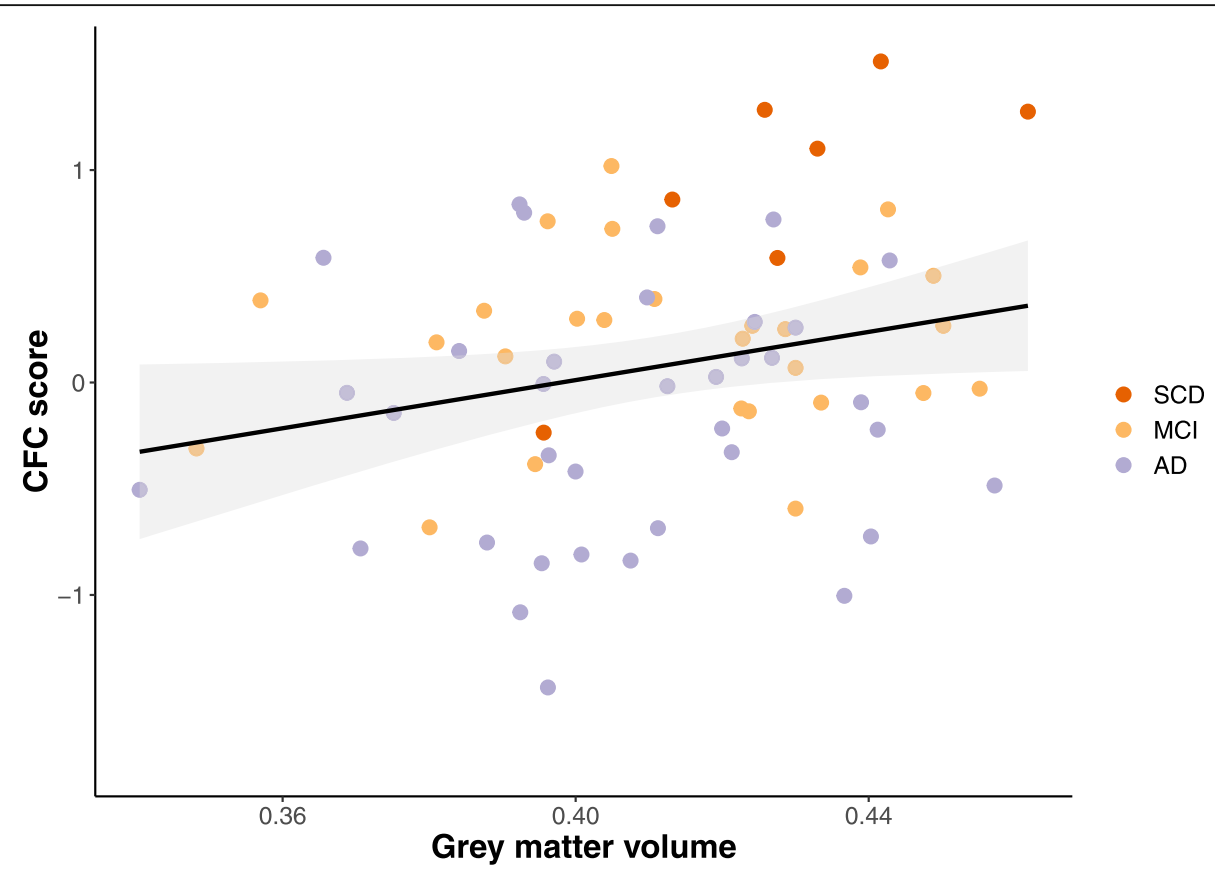

Fig. 3 Scatterplot displaying the correlation between the CFC score and gray matter volume (corrected for total intracranial volume)

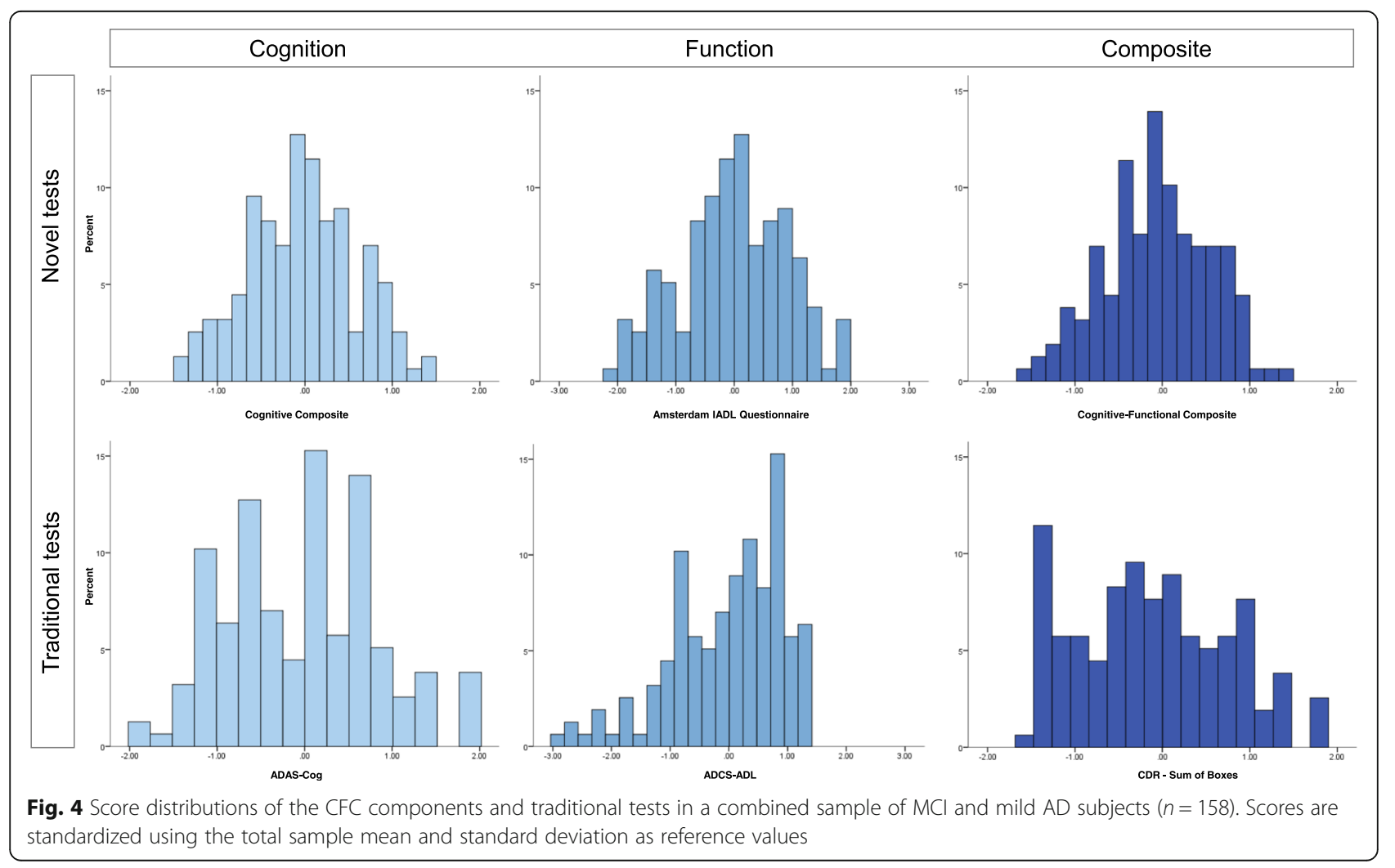


Table 4 Score ranges, percentiles, and distribution parameters for the traditional test scores and CFC scores

\begin{tabular}{|c|c|c|c|c|c|c|c|c|c|c|c|}
\hline & \multicolumn{2}{|c|}{ Score range } & \multicolumn{7}{|c|}{ Percentiles } & \multicolumn{2}{|c|}{ Distribution } \\
\hline & Original & Current sample & 5 & 10 & 25 & 50 & 75 & 90 & 95 & Skewness & Kurtosis \\
\hline ADAS-Cog & $0-80$ & $6-47$ & 14 & 16 & 19 & 26 & 30 & 35 & 40 & .236 & .217 \\
\hline ADCS-ADL & $0-78$ & $39-78$ & 47 & 55 & 60 & 68 & 73 & 75 & 77 & -.902 & .477 \\
\hline CDR-SB & $0-18$ & $0-12$ & .5 & .7 & 2.0 & 3.5 & 5.5 & 7.0 & 8.7 & .596 & .142 \\
\hline ADCOMS & $0-1.47$ & $.05-1.21$ & .10 & .15 & .28 & .43 & .61 & .86 & .99 & .620 & -.042 \\
\hline A-IADL-Q & N.A. & $-2.01-1.95$ & -1.71 & -1.32 & -.64 & .00 & .63 & 1.04 & 1.42 & -.091 & -.457 \\
\hline Cognitive Composite & N.A. & $-1.44-1.33$ & -1.13 & -.88 & -.52 & -0.04 & .40 & .81 & .96 & -.046 & -.445 \\
\hline CFC & N.A. & $-1.51-1.37$ & -1.14 & -.87 & -.42 & -0.04 & .38 & .78 & .88 & -.136 & -.440 \\
\hline
\end{tabular}

Abbreviations: ADAS-Cog Alzheimer's Disease Assessment Scale-Cognitive subscale, ADCOMS Alzheimer's Disease Composite Score, ADCS-ADL Alzheimer's Disease Cooperation Study-Activity of Daily Living, CDR-SB Clinical Dementia Rating Sum of Boxes, CFC cognitive-functional composite

illustrates that the CFC assesses a construct that is related to the underlying disease process. Taken together, these associations suggest that several clinically relevant aspects of the disease and its severity are captured by the CFC.
Quality for the target population was evaluated by inspecting range restrictions in scoring in our MCI and early dementia sample. This population largely corresponds with "stage 3 patients" as described in the recently proposed NIA-AA clinical staging scheme and
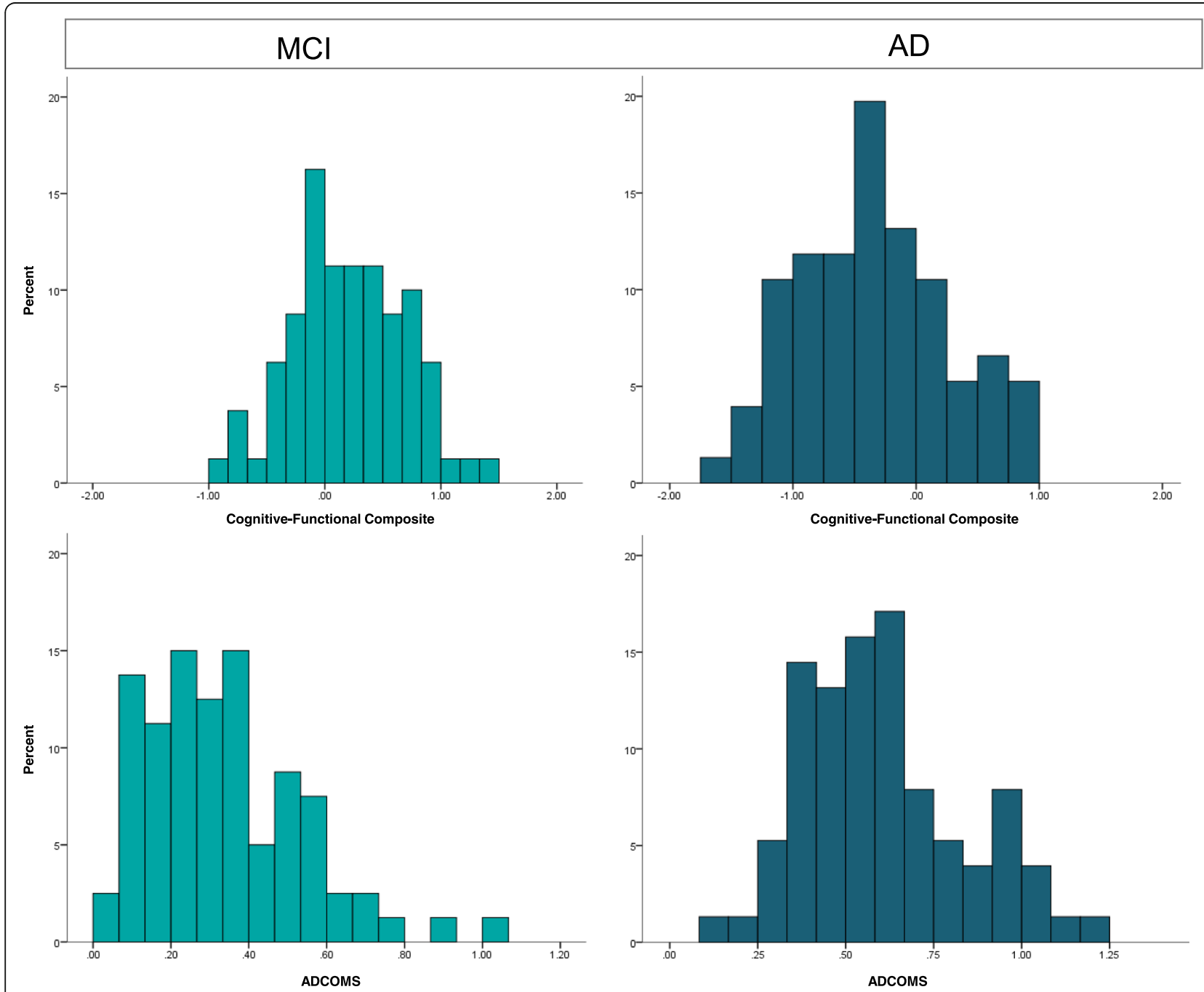

Fig. 5 Score distributions of the CFC and ADCOMS, separately for MCI $(n=80)$ and mild AD dementia $(n=78)$ 
aligned FDA guidance [11, 44]. We found that both the traditional tests and the recently designed ADCOMS showed ceiling effects in scoring in this stage, indicating that these participants showed a high level of functioning. These ceiling effects hamper the measurement of changes and especially the measurement of potential improvement due to treatment. In contrast, the score distributions of the CFC component were normally distributed, showing potential to indicate both decline and improvement with respect to baseline measurements. Therefore, these cross-sectional results support that the CFC is a promising measure with which to assess changes over time without exhibiting the limitations of traditionally used tests.

Several other endeavors have been undertaken to design and validate composite measures that are more appropriate to assess changes earlier clinical stages of $\mathrm{AD}$. These composites range from purely statistically driven $[27,45]$ to more theoretically based such as the preclinical Alzheimer's cognitive composite (PACC) [46] and the Alzheimer prevention initiative cognitive composite (APCC) [47]. The PACC and APCC focus on preclinical stages of $\mathrm{AD}$ and do not include a measure of daily function. However, measuring everyday functioning is highly relevant in the MCI and dementia stages, as evolving IADL problems are an important clinical hallmark in the transitional stage from $\mathrm{MCI}$ to dementia and predict a future decline in dementia [7]. The ADCOMS procedure includes a functional measure (CDR-SB), and the fact that previously changes were detected in a clinical trial [27] supports the idea that adding a functional measure advances a cognitive outcome measure. However, the ADCOMS selection has been largely driven by statistical considerations rather than its content, and therefore, its clinical relevance is as yet uncertain. It is our view that the CFC can contribute to the existing initiatives to improve cognitive measurement in the MCI and mild dementia stages, as its composition has been based on both theoretical constructs (i.e., the combination of cognition and IADL measure) and empirical research [17, 22].

There are some limitations to consider. First, not all $\mathrm{MCI}$ and $\mathrm{AD}$ participants had $\mathrm{AD}$ biomarkers available, and therefore, it is unknown whether they had AD pathology. However, in these circumstances, we relied on an extensive clinical assessment and excluded participants with other conditions that could have caused or contributed to the cognitive or functional symptoms. Second, some heterogeneity in our sample may have been caused by minor differences in the recruitment strategies employed across the centers as well as from including SCD and DLB participants. Consequently, our sample may not perfectly mirror the composition of an ideal clinical trial sample. It should also be noted that the SCD and DLB samples were relatively small and that the
CFC investigations in these groups were of explorative nature. This limits the interpretation of the CFC results in those groups. Additionally, the sample size of participants with an MRI scan available was relatively small, as this was not required for participation in our study. Therefore, we should be careful with interpreting these findings, particularly in the SCD group. A further limitation is that we have only investigated a single weighting method to create the CFC score, whereas it is likely that the optimal scoring method involves different weights for the different components. For example, our data showed that the memory component seems to be relatively easy compared to the EF component, which might be something that we need to account for when tracking the changes over time, in particular for the MCI group. Lastly, the fact that the CFC in its current composition focuses less on other domains than episodic memory and EF may limit its usefulness for measuring progression at more severe stages of dementia.

Strengths of this study include our construct validity approach, which is a unique aspect of the Catch-Cog study. Given the lack of a gold standard for "disease severity," we used different reference measures of disease severity to compare the CFC with, which led to converging evidence for the clinical relevance of the CFC. Additionally, we were able to perform a direct head-to-head comparison between the CFC and traditional tests, which, to our knowledge, has never been done in previous studies. The advantage of this is to reveal both the strengths and weaknesses of different clinical measurements. Furthermore, our investigation of the CFC in an independent, prospective cohort is an essential aspect of this study. Although the CFC consists of tests that have been validated as part of other test batteries and across several study cohorts, it cannot be assumed that all measures perform similarly in a novel composition. For example, tests may perform differently when assessed in a different test order. An independent validation of a novel composite measure such as the CFC measure is thus needed to enhance future implementation. We are currently assessing the CFC longitudinally in our CatchCog prospective study cohort, and we will investigate its sensitivity to change after 3, 6, and 12 months and compare its sensitivity with that of the traditional tests. This longitudinal data will also enable us to explore whether different weights for the subtests can improve the sensitivity to change over time, as well as whether different weights are useful when tracking a change in different diagnostic groups. For example, putting more weight on the cognitive parts and activities of daily living that decline relatively late in the disease course may enhance the use of the CFC to track progression in later disease stages. 


\section{Conclusions}

We demonstrated that the CFC has good construct validity and captures clinically relevant aspects of disease progression. We also showed its improved suitability for the target population as compared to traditional tests, as reflected by fewer range restrictions in scoring. These findings illustrate that the CFC has good potential to be a sensitive, clinically meaningful outcome measure. It is therefore better indicated for use to evaluate cognition and function as compared to traditional tests, as in line with the recent FDA recommendations. Ultimately, the CFC can yield a more accurate and useful measurement of clinically relevant changes, which will aid the monitoring of disease progression and evaluation of novel treatments.

\section{Additional file}

Additional file 1: MRI settings. (DOCX $13 \mathrm{~kb}$ )

\begin{abstract}
Abbreviations
AD: Alzheimer's disease; ADAS-Cog: Alzheimer's Disease Assessment ScaleCognitive subscale; ADCOMS: Alzheimer's Disease Composite Score; ADCSADL: Alzheimer's Disease Cooperative Study-Activities of Daily Living; AES: Apathy Evaluation Scale; A-IADL-Q: Amsterdam IADL Questionnaire; Catch-Cog: Capturing Changes in Cognition; CC: Cognitive composite; CDRSB: Clinical Dementia Rating scale Sum of Boxes; CFA: Confirmatory factor analysis; CFC: Cognitive-functional composite; CFI-SP: Cognitive Function Instrument - study partner version; CFI: Comparative Fit Index; CFT: Category fluency test; COWAT: Controlled Oral Word Association Test; CSF: Cerebrospinal fluid; DLB: Dementia with Lewy bodies; DSST: Digit symbol substitution test; EF: Executive functioning; EM: Episodic memory; FLAIR: Fluid-attenuated inversion recovery; GM: Gray matter;

IADL: Instrumental activities of daily living; IRT: Item response theory; MCl: Mild cognitive impairment; MMSE: Mini-Mental State Examination; MRI: Magnetic resonance imaging; QoL: Quality of life; RMSEA: Root mean squares of error approximation; SCD: Subjective cognitive decline; SRMR: Standardized root mean square residual; TIV: Total intracranial volume; WM: White matter; WM: Working memory; ZBI: Zarit Burden Inventory
\end{abstract}

\section{Acknowledgements}

The authors would like to thank Mandy Ter Haar, Larissa Masselink, Judith Meurs, Anne Brunner, Mieke Geertsma, Nina Schimmel, llya de Groot, Judy van Hemmen, Kate Forsyth, Sarah Gregory, Neil Fullerton, Clare Dolan, and Matthew Hunter for their help with the data collection. Additionally, we would like to acknowledge Stichting Buytentwist for their support. Research of the Alzheimer Center Amsterdam is part of the neurodegeneration research program of Amsterdam Neuroscience. The Alzheimer Center Amsterdam is supported by Alzheimer Nederland and Stichting VUmc Fonds.

\section{Funding}

The present study is supported by a grant from Memorabel (grant no. 733050205), which is the research program of the Dutch Deltaplan for Dementia.

\section{Availability of data and materials}

All data used and/or analyzed during the current study are available from the corresponding author on reasonable request.

\section{Authors' contributions}

RJJ, JEH, and SAMS designed the study, performed the statistical analyses, interpreted the results, and drafted the manuscript. PRL, SI, RV, RAJD, FJJ, EMO, AA, CWR, and PS helped interpret the results and edit the manuscript. RJJ wrote the final version of the manuscript. All authors read and approved the final manuscript.

\section{Ethics approval and consent to participate}

The Medical Ethical Committee of the VU University Medical Center approved the study for all Dutch centers. The South East Scotland Research Ethics Committee approved the study for the Scottish site. All participants and study partners provided written and oral informed consent for the use of their data for research purposes.

\section{Consent for publication}

Not applicable

\section{Competing interests}

RJJ, PRL, RV, RAJD, FJJ, EMO, and CWR declare that they have no competing interests. In the past 2 years, JEH has received honoraria and paid consultancy from 23andMe, Abbvie, A2Q, AlzCure, Amgen, Anavex, Aptinyx, Astellas, AstraZeneca, Avraham, Axon, Axovant, Biogen, Boehringer Ingelheim, Bracket, Catenion, Cognition Therapeutics, CRF Health, Curasen, DeNDRoN, Enzymotec, Eisai, Eli Lilly, GfHEu, Heptares, Johnson \& Johnson, Kaasa Health, Lysosome Therapeutics, Lundbeck, Merck, MyCognition, Mind Agilis, Neurocog, Neurim, Neuroscios, Neurotrack, Novartis, Nutricia, Pfizer, Pricespective, Probiodrug, Regeneron, Rodin Therapeutics, Roche, Sanofi, Servier, Shire, and Takeda \& vTV Therapeutics. SI received support from the EU/EFPIA Innovative Medicines Initiative Joint Undertaking EPAD grant agreement no. 115736. AA has received speaker fees from Lundbeck. PS has acquired grant support (for the institution) from GE Healthcare and Piramal. In the past 2 years, he has received consultancy/speaker fees (paid to the institution) from Novartis, Probiodrug, Biogen, Roche, and EIP Pharma, LLC. BMT receives grant support from ZonMW. SAMS is supported by grants from JPND and Zon-MW and has provided consultancy services in the past 2 years for Lundbeck. All funds were paid to her institution.

\section{Publisher's Note}

Springer Nature remains neutral with regard to jurisdictional claims in published maps and institutional affiliations.

\section{Author details \\ ${ }^{1}$ Alzheimer Center Amsterdam, Department of Neurology, Amsterdam Neuroscience, Amsterdam UMC, Vrije Universiteit Amsterdam, Amsterdam, The Netherlands. ${ }^{2}$ Metis Cognition Ltd, Park House, Kilmington Common, Wiltshire, UK. ${ }^{3}$ Institute of Psychiatry, Psychology \& Neuroscience, King's College London, London, UK. ${ }^{4}$ Department of Radiology and Nuclear Medicine Amsterdam UMC, Vrije Universiteit Amsterdam, Amsterdam, The Netherlands. ${ }^{5}$ Department of Geriatrics, Spaarne Gasthuis, Haarlem, The Netherlands. ${ }^{6}$ Department of Neurology, Erasmus Medical Center, Rotterdam, The Netherlands. ${ }^{7}$ Department of Biomedical Sciences, University Medical Center Groningen, University of Groningen, Groningen, The Netherlands. ${ }^{8}$ Department of Health and Social Work, University of Applied Sciences Windesheim, Zwolle, The Netherlands. ${ }^{9}$ Centre for Dementia Prevention, University of Edinburgh, Edinburgh, UK. ${ }^{10}$ Department of Epidemiology \& Biostatistics, Amsterdam UMC, Vrije Universiteit Amsterdam, Amsterdam, The Netherlands.}

Received: 29 January 2019 Accepted: 28 April 2019

Published online: 15 May 2019

\section{References}

1. Scheltens P, et al. Alzheimer's disease. Lancet. 2016;388(10043):505-17.

2. Albert MS, et al. The diagnosis of mild cognitive impairment due to Alzheimer's disease: recommendations from the National Institute on Aging-Alzheimer's Association workgroups on diagnostic guidelines for Alzheimer's disease. Alzheimers Dement. 2011;7(3):270-9.

3. Rosen WG, Mohs RC, Davis KL. A new rating scale for Alzheimer's disease. Am.J.Psychiatry. 1984;141(11):1356-64.

4. Karin A, et al. Psychometric evaluation of ADAS-Cog and NTB for measuring drug response. Acta Neurol Scand. 2014;129(2):114-22

5. Kueper JK, Speechley M, Montero-Odasso M. The Alzheimer's Disease Assessment Scale-Cognitive Subscale (ADAS-Cog): modifications and responsiveness in pre-dementia populations. A narrative review. J Alzheimers Dis. 2018;63(2):423-44.

6. Sikkes SA, et al. A systematic review of Instrumental Activities of Daily Living scales in dementia: room for improvement. J Neurol Neurosurg Psychiatry. 2009;80(1):7-12. 
7. Jekel $\mathrm{K}$, et al. Mild cognitive impairment and deficits in instrumental activities of daily living: a systematic review. Alzheimers Res Ther. 2015;7(1):17.

8. Cano SJ, et al. The ADAS-Cog in Alzheimer's disease clinical trials: psychometric evaluation of the sum and its parts. J Neurol Neurosurg Psychiatry. 2010;81(12):1363-8.

9. Snyder PJ, et al. Assessing cognition and function in Alzheimer's disease clinical trials: do we have the right tools? Alzheimers Dement. 2014;10(6): 853-60.

10. Kozauer N, Katz R. Regulatory innovation and drug development for earlystage Alzheimer's disease. N Engl J Med. 2013;368(13):1169-71.

11. Food and Drug Administration. Early Alzheimer's disease: developing drugs for treatment. Guidance for industry. 2018.

12. European Medicines Agency. Guideline on the clinical investigation of medicines for the treatment of Alzheimer's disease. 2018.

13. Vellas B, et al. Endpoints for trials in Alzheimer's disease: a European task force consensus. Lancet Neurol. 2008;7(5):436-50.

14. Lemstra AW, et al. Concomitant AD pathology affects clinical manifestation and survival in dementia with Lewy bodies. J Neurol Neurosurg Psychiatry. 2017;88(2):113-8.

15. McKeith IG, et al. Diagnosis and management of dementia with Lewy bodies: fourth consensus report of the DLB consortium. Neurology. 2017; 89(1):88-100.

16. Jutten RJ, et al. A composite measure of cognitive and functional progression in Alzheimer's disease: design of the Capturing Changes in Cognition study. Alzheimers Dement. 2017;3(1):130-8.

17. Harrison J, et al. Validation of a novel cognitive composite assessment for mild and prodromal Alzheimer's disease. Alzheimers Dement. 2013;9(4):P661.

18. Sikkes SA, et al. A new informant-based questionnaire for instrumental activities of daily living in dementia. Alzheimers Dement. 2012;8(6):536-43.

19. Sikkes SA, et al. Validation of the Amsterdam IADL Questionnaire@, a new tool to measure instrumental activities of daily living in dementia. Neuroepidemiology. 2013:41(1):35-41.

20. Sikkes SA, et al. Assessment of instrumental activities of daily living in dementia: diagnostic value of the Amsterdam Instrumental Activities of Daily Living Questionnaire. J Geriatr Psychiatry Neurol. 2013;26(4):244-50.

21. Jutten RJ, et al. Detecting functional decline from normal aging to dementia: development and validation of a short version of the Amsterdam IADL Questionnaire. Alzheimers Dement. 2017:8:26-35.

22. Koster $\mathrm{N}$, et al. The sensitivity to change over time of the Amsterdam IADL Questionnaire@. Alzheimers Dement. 2015;11(10):1231-40.

23. Jutten RJ, et al. A novel cognitive-functional composite measure to detect changes in early Alzheimer's disease: test-retest reliability and feasibility. Alzheimers Dement. 2018;10:153-60.

24. de Vet HCW, et al. Measurement in medicine. New York: Cambridge University Press; 2011.

25. Hughes $C P$, et al. A new clinical scale for the staging of dementia. $\mathrm{Br}$ J Psychiatry. 1982;140:566-72.

26. Galasko D, et al. An inventory to assess activities of daily living for clinical trials in Alzheimer's disease. The Alzheimer's Disease Cooperative Study. Alzheimer DisAssocDisord. 1997;11(Suppl 2):S33-9.

27. Wang J, et al. ADCOMS: a composite clinical outcome for prodroma Alzheimer's disease trials. J Neurol Neurosurg Psychiatry. 2016;87(9):993-9.

28. Jessen $\mathrm{F}$, et al. A conceptual framework for research on subjective cognitive decline in preclinical Alzheimer's disease. Alzheimers Dement. 2014;10(6):844-52.

29. McKhann GM, et al. The diagnosis of dementia due to Alzheimer's disease: recommendations from the National Institute on Aging-Alzheimer's Association workgroups on diagnostic guidelines for Alzheimer's disease. Alzheimers Dement. 2011;7(3):263-9.

30. Yesavage JA, et al. Development and validation of a geriatric depression screening scale: a preliminary report. J Psychiatr Res. 1983;17(1):37-49.

31. Lezak MD. Neuropsychological assessment. USA: Oxford University Press; 2004.

32. Embretson SE, Reise SP. Item response theory. Mahwah, New Jersey: Psychology Press; 2013.

33. Folstein MF, Folstein SE, McHugh PR. "Mini-mental state". A practical method for grading the cognitive state of patients for the clinician. J Psychiatr Res. 1975;12(3):189-98.

34. Williams MM, et al. Progression of Alzheimer's disease as measured by Clinical Dementia Rating Sum of Boxes scores. Alzheimers Dement. 2013:9(1 Suppl):S39-44.

35. Amariglio $R E$, et al. Tracking early decline in cognitive function in older individuals at risk for Alzheimer disease dementia: the Alzheimer's disease cooperative study cognitive function instrument. JAMA Neurol. 2015;72(4): 446-54.

36. Logsdon RG, et al. Assessing quality of life in older adults with cognitive impairment. Psychosom.Med. 2002;64(3):510-9.

37. Bedard $\mathrm{M}$, et al. The Zarit Burden Interview: a new short version and screening version. Gerontologist. 2001;41(5):652-7.

38. Marin RS, Biedrzycki RC, Firinciogullari S. Reliability and validity of the Apathy Evaluation Scale. Psychiatry Res. 1991;38(2):143-62.

39. Kline RB. Principles and practice of structural equation modeling. New York: Guilford publications; 2015.

40. Harrison J, et al. Cognition in $\mathrm{MCl}$ and Alzheimer's disease: baseline data from a longitudinal study of the NTB. Clin Neuropsychol. 2014;28(2):252-68.

41. Prinsen CA, et al. How to select outcome measurement instruments for outcomes included in a "Core Outcome Set" - a practical guideline. Trials. 2016:17(1):449.

42. Jack $\mathrm{CR}$, et al. Brain atrophy rates predict subsequent clinical conversion in normal elderly and amnestic MCI. Neurology. 2005;65(8):1227-31.

43. Ten Kate M, et al. Secondary prevention of Alzheimer's dementia: neuroimaging contributions. Alzheimers Res Ther. 2018;10(1):112.

44. Jack CR Jr, et al. NIA-AA Research Framework: toward a biological definition of Alzheimer's disease. Alzheimers Dement. 2018;14(4):535-62.

45. Burnham SC, et al. Novel statistically-derived composite measures for assessing the efficacy of disease-modifying therapies in prodromal Alzheimer's disease trials: an AIBL study. J Alzheimers Dis. 2015:46(4):1079-89.

46. Donohue MC, et al. The preclinical Alzheimer cognitive composite: measuring amyloid-related decline. JAMA Neurol. 2014;71(8):961-70.

47. Langbaum JB, et al. An empirically derived composite cognitive test score with improved power to track and evaluate treatments for preclinical Alzheimer's disease. Alzheimers Dement. 2014;10(6):666-74.
Ready to submit your research? Choose BMC and benefit from:

- fast, convenient online submission

- thorough peer review by experienced researchers in your field

- rapid publication on acceptance

- support for research data, including large and complex data types

- gold Open Access which fosters wider collaboration and increased citations

- maximum visibility for your research: over $100 \mathrm{M}$ website views per year

At BMC, research is always in progress.

Learn more biomedcentral.com/submissions 